\title{
Isolated attosecond pulses using a detuned second-harmonic field
}

\author{
Hamed Merdji, ${ }^{1,2, *}$ Thierry Auguste, ${ }^{1}$ Willem Boutu, ${ }^{1}$ J.-Pascal Caumes, ${ }^{1}$ Bertrand Carré, ${ }^{1}$ \\ Thomas Pfeifer, ${ }^{3,4}$ Aurélie Jullien, ${ }^{3,4}$ Daniel M. Neumark, ${ }^{3,4}$ and Stephen R. Leone ${ }^{3,4}$ \\ ${ }^{1}$ Service des Photons Atomes et Molécules, Commissariat à l'Energie Atomique, Centre d'Etudes de Saclay, \\ Bâtiment 522, 91191 Gif sur Yvette, France \\ ${ }^{2}$ Stanford PULSE Center, SLAC, Menlo Park, California 94025, USA \\ ${ }^{3}$ Departments of Chemistry and Physics, University of California, Berkeley, California 94720, USA \\ ${ }^{4}$ Chemical Sciences Division, Lawrence Berkeley National Laboratory, Berkeley, California 94720, USA \\ *Corresponding author: merdji@drecam.cea.fr
}

\begin{abstract}
Calculations are presented for the generation of an isolated attosecond pulse in a multicycle two-color strong-field regime. We show that the recollision of the electron wave packet can be confined to half an optical cycle using pulses of up to $40 \mathrm{fs}$ in duration. The scheme is proven to be efficient using two intense beams, one producing a strong field at $\omega$ and the other a strong field detuned from $2 \omega$. The slight detuning $\delta \omega$ of the second harmonic is used to break the symmetry of the electric field over many optical cycles and provides a coherent control for the formation of an isolated attosecond pulse.
\end{abstract}

High-order harmonics generation (HHG) of intense laser pulses in gases is attracting much attention due to both fundamental and applied interests. One of the most attractive properties of the HHG source is the generation of attosecond pulses. This field has been growing spectacularly (for reviews see $[1,2]$ ). Trains of attosecond pulses are naturally produced in the HHG process, whereas the generation of a single or isolated attosecond pulse is more challenging. Isolated attosecond pulses have been, however, achieved using absolute phase-stabilized few-optical-cycle state-of-the art laser systems. The methods rely either on the laser intensity [3] or ellipticity (polarization state) $[4,5]$ dependencies of the harmonic emission. Another approach involves control of the wave packet recollision using a two-color field. Proposals based on a mixing scheme of a strong fundamental field and a weak [6] or strong [7] second-harmonic field have been investigated computationally. Experimental indications of an isolated attosecond pulse have been observed in the latter scheme explored experimentally. These schemes are very promising but require very short pulses $(<12 \mathrm{fs})$ except if harmonic heterodyning with a weak lower frequency field is used [8], which is, however, more difficult to produce experimentally than the second harmonic.

In this Letter, we propose to generate single attosecond pulses in the long optical cycle regime by mixing the fundamental field to its detuned second harmonic. To illustrate the principle, we perform onedimension quantum mechanical calculations using an argon atom with a smoothed Coulomb potential. Our approach is based on the numerical solution of the time-dependent Schrödinger equation. The calculated attosecond time structure is shown in Fig. 1a when only the fundamental $\left(\omega_{1}\right)$ is present. The laser intensity is constant. We see that an attosecond pulse is emitted every $1 / 2$ optical cycle. This reflects the symmetry of the HHG process. In Fig. $1 \mathrm{~b}$ we perform the same calculations but add an intense spectrally detuned second-harmonic field. The detuning is chosen so that $\omega_{2}=\omega_{1}(2+1 / N)$, with $N=5$. Due to the new periodicity of the electric field we show that an attosecond pulse is now emitted every 5/2 optical cycle. This property of HHG can be used to isolate a single attosecond pulse. Indeed, if the laser envelope duration is of the same order as (or a few times) the delay between two consecutive attosecond pulses, the strong intensity dependence of the HHG process will allow the isolation of a single attosecond pulse.

This is shown in Fig. 2 with $N=5.5$ and with an envelope of ten optical cycles for both the fundamental and the detuned second-harmonic fields. The timedependent laser pulse envelope has a $\sin ^{2}\left(\pi t / 2 \tau_{L}\right)$ shape, where $t$ is time and $\tau_{L}$ the FWHM. The intensities of the fundamental and the second-harmonic fields are $2 \times 10^{14}$ and $10^{14} \mathrm{~W} / \mathrm{cm}^{2}$, respectively. The electric field symmetry is strongly broken so that the recollision of the most energetic part of the wave packet is now confined to half an optical cycle within the full envelope. The calculation shows an isolated attosecond pulse (80 as) with a large spectral bandwidth of $24 \mathrm{eV}$ centered at $49 \omega$ (see below). In the simulation the strong field regime produced by the two colors plays a significant role. The population of the ground state is plotted in Fig. 2. The ionization is taken directly from the resolution of the Schrödinger equation. The ground state is totally depleted after the maximum of the electric field so that no harmonic emission is allowed on the trailing edge of the pulse. It should be noted that this effect has recently been proposed to generate a single attosecond pulse [9]. In the present case the ground state population is still high (30\%) just before the emission of the attosecond 


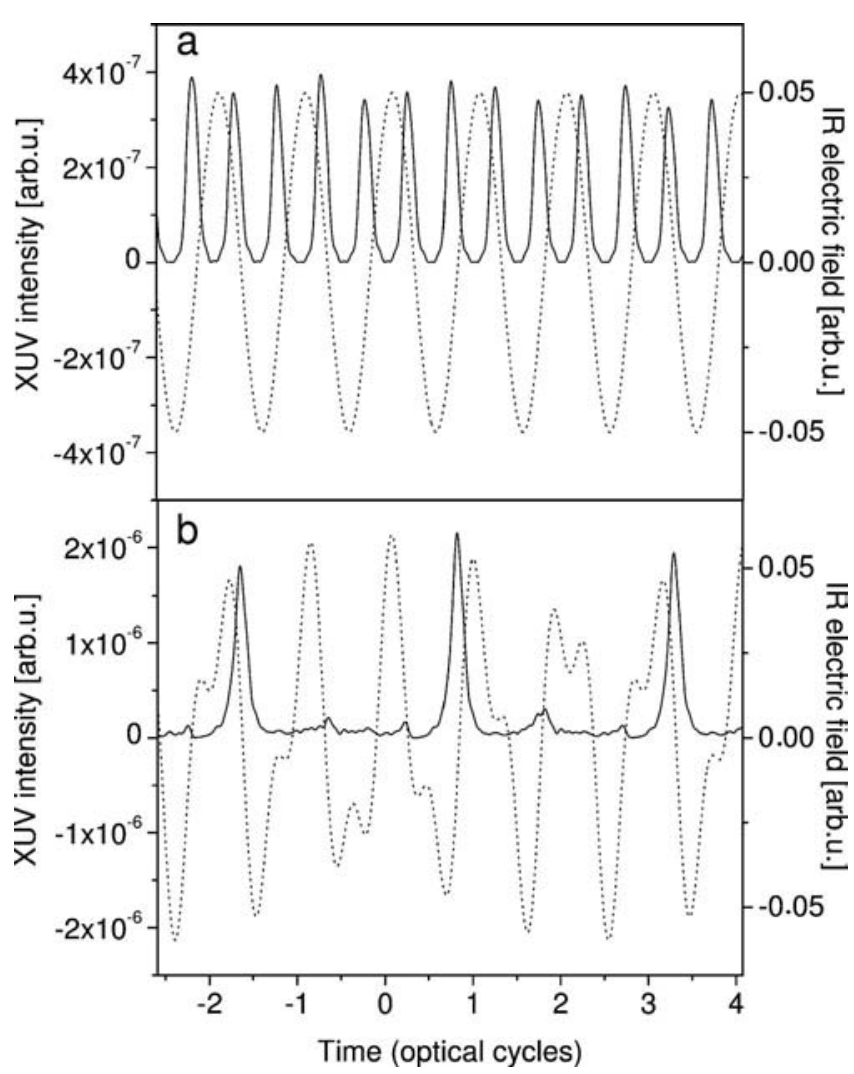

Fig. 1. Time profiles with only the a, fundamental $(800 \mathrm{~nm}) \omega_{1}$ and $\mathrm{b}$, when mixing the fundamental and its detuned second harmonic $\omega_{2}=2.2 \omega_{1}$. The laser intensity is constant. The relative phase between the two fields is $0.64 \pi$. Dotted curves: total generating electric field. Solid curves: calculations of the harmonic emission in argon using a numerical filter centered at $26 \omega_{1}$ with a $20 \mathrm{eV}$ bandwidth.

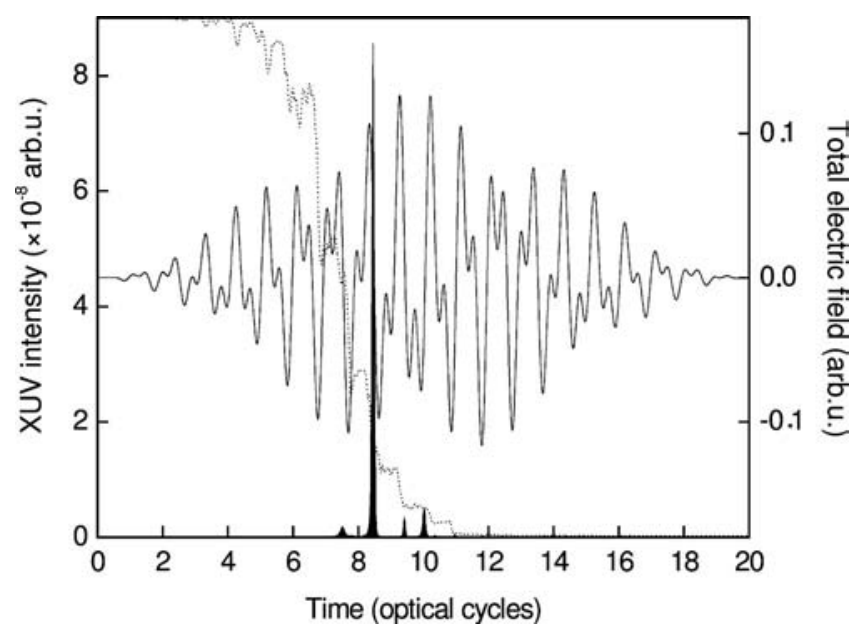

Fig. 2. Driving electric field of the two-color detuned scheme (solid curve) with $\omega_{2}=2.18 \omega_{1}$. Calculation of the harmonic emission in argon (shaded area curve) using a numerical spectral filter between from $41 \omega_{1}$ to above the cutoff (see text). The fundamental field is centered at $800 \mathrm{~nm}$. Both fields have an envelope of ten optical cycles ( $27 \mathrm{fs}, \mathrm{FWHM})$. The relative phase of the two fields is equal to 0 . Dotted curve: normalized population of the ground state. The population is evolving from 1 at $t=0$ to 0 at $t$ $=20$ optical cycles. pulse so that the gating method is quite efficient. Calculations (not shown here) at lower intensity ( $10^{14}$ and $5 \times 10^{13} \mathrm{~W} / \mathrm{cm}^{2}$ for the fundamental and the second harmonic, respectively) indicate that when the medium is not depleted we can obtain secondary attosecond pulses in the trailing edge of the pulse. The phase difference between the two colors is also a very important parameter and should be adjusted with interferometric stability in an experiment. Our calculations show that a phase change from 0 to $\pi / 4$ affects the time profile of the radiation. Indeed a second attosecond pulse is emitted with $\sim 40 \%$ of the intensity of the main pulse. The method also relies on the phase slippage between the two colors in the generating medium, which should be kept below $\pi / 10$. This can be matched, for example, in a millimeter length, few tens of torr of argon gas medium.

The relative phase of the two pulses also plays a role in controlling the wave packet dynamics. Indeed the two-color electric fields add coherently so that the most energetic single wave packet recollision is observed for the highest field acceleration. This can be adjusted by the relative phase of the fields so that the recollision occurs close to the maximum of the optical pulse envelope. In that sense this technique is more efficient than the intensity [3] or polarization gating $[4,5]$ methods where no coherent control on the attosecond electron wave packet properties is applied. The constructive coherent sum of the fields leads to a much higher electron recollision kinetic energy so that an extension of the high harmonic plateau occurs. This is illustrated in Fig. 3 where the spectrum is plotted for the single color and the two-colordetuned schemes. The cutoff is significantly extended from harmonic 39 to harmonic 57. This is consistent with the additional kinetic energy of the returning electron wave packet provided by the detuned second-harmonic strong field. We note that in the large extended plateau region the spectrum shows less modulation than the single color scheme. This is the region where the numerical spectral filter is applied to isolate the attosecond pulse.

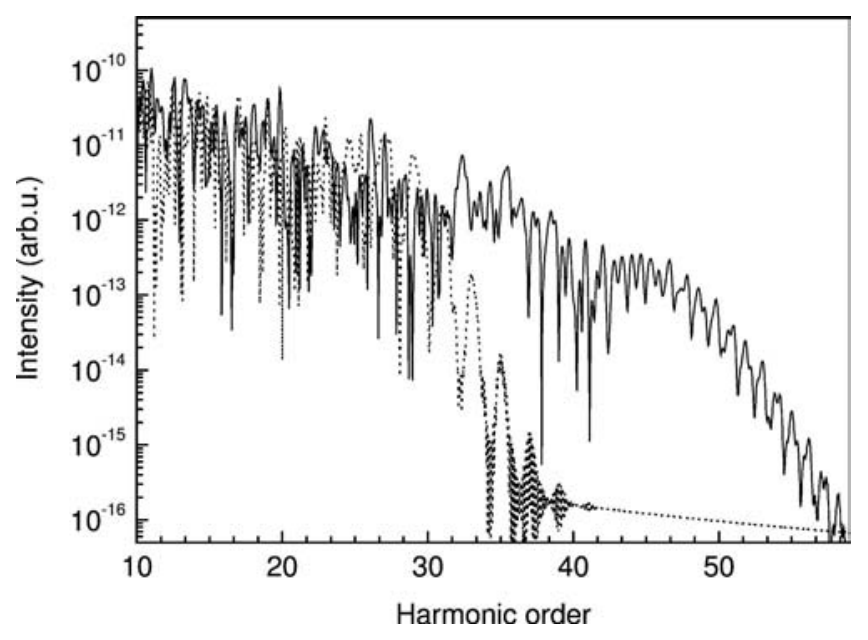

Fig. 3. Total harmonic spectra extracted from the quantum-mechanical calculations in argon shown in Fig. 2 (same parameters). Dotted curve: spectra generated using only the $\omega_{1}$ field. Solid curve: spectra generated in the mixing scheme with $\omega_{1}$ and $\omega_{2}=2.18 \omega_{1}$. 
We propose now to extend the technique to longer laser pulse duration available in most laboratories. Figure 4 shows quantum-mechanical calculations in argon using 15 optical cycles pulses (40 fs). The results have been obtained after optimizing the detuning and the phase difference between the two beams. The best case shown here is $N=5$ and $\phi=0.64 \pi$. The total electric field exhibits a clear modulation of the envelope with a period of 5 optical cycles and the inset shows that the intraoptical cycle symmetry is broken. The calculations reveal a high-contrast isolated attosecond pulse generated just before the maximum of the laser envelope. The small satellite pulses exhibits intensities below $5 \%$ of the main peak.

Here, the optimization of the parameters (dephasing and detuning) of isolated attosecond pulses has been done manually. However these parameters can be adjusted in an experiment by utilizing closed loop quantum control of the attosecond pulse generation. These two parameters can be inserted into an evolutionary algorithm [10] in order to shape the attosecond pulse structure. We also note that the method can use a detuning either of the second harmonic or of the fundamental field; the symmetry will be broken in either case. The detuning of the $800 \mathrm{~nm}$ wavelength is easier to produce using, for example, nonlinear phase modulation effects. The technique can be implemented on high energy lasers so that controllable single attosecond pulses in the microjoule range can be generated.

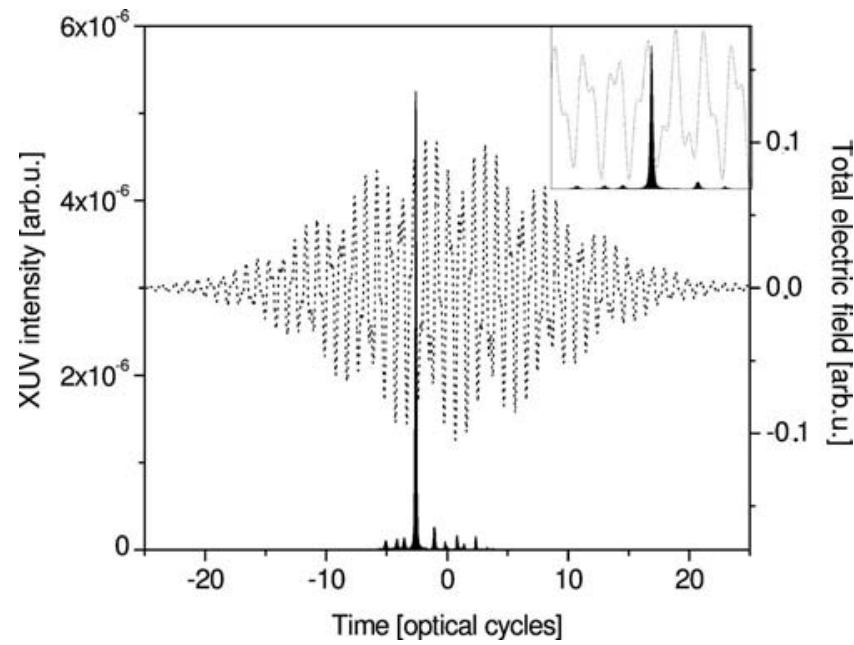

Fig. 4. Calculations of the attosecond pulses produced with the two-color mixing scheme for $\omega_{1}$ and $\omega_{2}=2.2 \omega_{1}$. Both driving fields have an envelope of 15 optical cycles (FWHM). The detuned-second-harmonic field has an additional phase of $\phi=0.64 \pi$. Dotted curve: total generating electric field. Shaded area curve: attosecond time structure of the harmonic emission. The inset shows the total electric field and the isolated attosecond pulse over a few-opticalcycle window.
Finally the method can be of general use in other strong field experiments. In high harmonic generation we have shown that a symmetry-broken strong field provides a way to generate isolated attosecond pulses with long optical pulses. Another application can be the possibility of controlling the recollision probability of a wave packet in a pump-probe experiment. The two fields and their relative properties can be tuned to "guide" a single attosecond wave packet with a controllable kinetic energy back toward the ground state. This could add considerable flexibility in the application of a wave packet as a probe of a molecular system $[11,12]$.

This research was supported by the New Emerging Science and Technology European contract TUIXS (NEST-012843), by the U.S. Department of Energy, through the Stanford PULSE Center and a MURI program from the Air Force Office of Scientific Research, contract FA9550-04-1-0242. Portions of the laboratory at Berkeley were supported by the Director, Office of Science, Office of Basic Energy Sciences, of the U.S. Department of Energy under contract DE-AC02-05CH11231. T. Pfeifer acknowledges support of a Feodor Lynen Fellowship of the Alexander von Humboldt Foundation.

\section{References}

1. P. Agostini and L. F. DiMauro, Rep. Prog. Phys. 67, 813 (2004).

2. P. B. Corkum and F. Krausz, Nat. Phys. 3, 381 (2007).

3. M. Hentschel, R. Kienberger, C. Spielmann, G. A. Reider, N. Miloševi'c, T. Brabec, P. B. Corkum, U. Heinzmann, M. Drescher, and F. Krausz, Nature 414, 509 (2001).

4. M. Ivanov, P. B. Corkum, T. Zuo, and A. Bandrauk, Phys. Rev. Lett. 74, 2933 (1995).

5. I. J. Sola, E. Mevel, L. Elouga, E. Constant, V. Strelkov, L. Poletto, P. Villoresi, E. Benedetti, J.-P. Caumes, S. Stagira, C. Vozzi, G. Sansone, and M. Nisoli, Nat. Phys. 2, 319 (2006).

6. T. Pfeifer, L. Gallmann, M. J. Abel, D. M. Neumark, and S. R. Leone, Opt. Lett. 31, 975 (2006).

7. Y. Oishi, M. Kaku, A. Suda, F. Kannari, and K. Midorikawa, Opt. Express 14, 7230 (2006).

8. T. Pfeifer, L. Gallmann, M. J. Abel, P. M. Nagel, D. M. Neumark, and S. R. Leone, Phys. Rev. Lett. 97, 163901 (2006).

9. W. Cao, P. Lu, P. Lan, X. Wang, and G. Yang, Phys. Rev. A 74, 063821 (2006).

10. R. Bartels, S. Backus, E. Zeek, L. Misoguti, G. Vdovin, I. P. Christov, M. M. Murnane, and H. C. Kapteyn, Nature 406, 164 (2000).

11. S. Baker, J. S. Robinson, C. A. Haworth, H. Teng, R. A. Smith, C. C. Chirila, M. Lein, J. W. G. Tisch, and J. P. Marangos, Science 312, 424 (2006).

12. J. Itatani, J. Levesque, D. Zeidler, Hiromichi Niikura, H. Pepin, J. C. Kieffer, P. B. Corkum, and D. M. Villeneuve, Nature 432, 7019 (2004). 